\title{
LA CERAMICA FENICIA DA NURAGHE S'URACHI E DAL villaggio di Su Padrigheddu (SAn Vero Milis, SARDEGNA): ASPETTI CRONOLOGICI E FUNZIONALI
}

\author{
ANDREA RoPPA \\ School of Archaeology and Ancient History \\ University of Leicester
}

Recibido: 4/02/2015

Revisado: 26/02/2015
Aceptado: $18 / 03 / 2015$

Publicado: 30/05/2015

\section{RiASSUNTO}

Il centro indigeno ubicato presso il nuraghe S'Urachi e l'adiacente villaggio di Su Padrigheddu, nella Sardegna centro-occidentale, fu abitato senza soluzione di continuità fra la tarda età del Bronzo e la prima età romana. Ricerche condotte presso i due siti hanno restituito un cospicuo lotto di materiali di tipologia fenicia, databili fra l'VIII e il VI sec. a.C. Nel presente articolo, mediante l'analisi delle caratteristiche cronologiche e funzionali della ceramica fenicia, in particolare anfore e ceramica da cucina e da mensa, si vuole porre in evidenza come la distribuzione di ceramica fenicia a S'Urachi e Su Padrigheddu incrementò in modo sostanziale dalla metà del VII sec. a.C. Mentre una cospicua percentuale dei materiali è rappresentata da frammenti di anfore, probabilmente importate dai vicini centri coloniali sulle sponde del golfo di Oristano, una parte significativa di ceramica di tipologia fenicia è composta da manufatti da cucina di produzione locale. Si suggerisce che questa categoria funzionale sia da mettere in relazione con pratiche culinarie tradizionali di un gruppo di individui di estrazione culturale fenicia, che giunsero a S'Urachi e Su Padrigheddu dai vicini centri coloniali e Tharros e/o Othoca.

\section{Parole chave}

Fenicio; Nuragico; Sardegna; Età del Ferro; Consumo Ceramico.

\section{ABstRact}

The indigenous site at nuraghe S'Urachi and the adjacent village at the site at Su Padrigheddu in west-central Sardinia were continuously settled between the Late Bronze age and the early Roman period. Research at both sites has yielded a significant assemblage of typologically Phoenician pottery, dating between the $8^{\text {th }}$ and $6^{\text {th }} \mathrm{c}$. BC. By focusing on chronological and functional features of Phoenician pottery, namely amphorae, cooking and table ware, my aim in this paper is to show that the distribution of Phoenician ceramics at S'Urachi and Su Padrigheddu dramatically increased from the mid- $7^{\text {th }}$ century BC. While a substantial percentage of the assemblage is made up of amphora sherds, which were imported from the nearby Phoenician colonial settlements located on the shores of the Gulf of Oristano, a significant amount of typologically Phoenician pottery is composed of locally made cooking ware. It is suggested that this functional category of pottery is related to the culinary, traditional practices of a group of individuals of Phoenician background, who moved from the nearby colonial settlements of Tharros and/or Othoca and settled at S'Urachi and Su Padrigheddu.

KEYWORDS

Phoenician; Nuragic; Sardinia; Iron Age; Pottery Consumption 


\section{INTRODUZIONE}

L'imponente complesso di nuraghe S'Urachi (o S’Uraki) e l'adiacente villaggio di Su Padrigheddu, nella Sardegna centro-occidentale, furono insediati senza soluzione di continuità fra la tarda età del Bronzo - probabile momento di costruzione del nuraghe - e la prima epoca romana. Le ricerche presso questi due siti hanno restituito un significativo lotto di materiali, le cui similarità funzionali e cronologiche permettono di identificare S'Urachi e $\mathrm{Su}$ Padrigheddu come un unico grande insediamen- to attivo lungo tutto il I millennio a.C. Tra questi materiali, quelli di tipologia fenicia che sono l'oggetto di questo contributo, forniscono importanti informazioni sia sui rapporti commerciali fra la locale popolazione nuragica e gruppi di Fenici nel corso dell'età del Ferro, sia sulla ricezione e consumo di manufatti non tradizionali in una comunità indigena sarda (figura 1).

Nel presente lavoro, si vuole porre l'attenzione sul tipo di materiale ceramico fenicio recuperato presso questi siti, per comprendere le fasi crono-

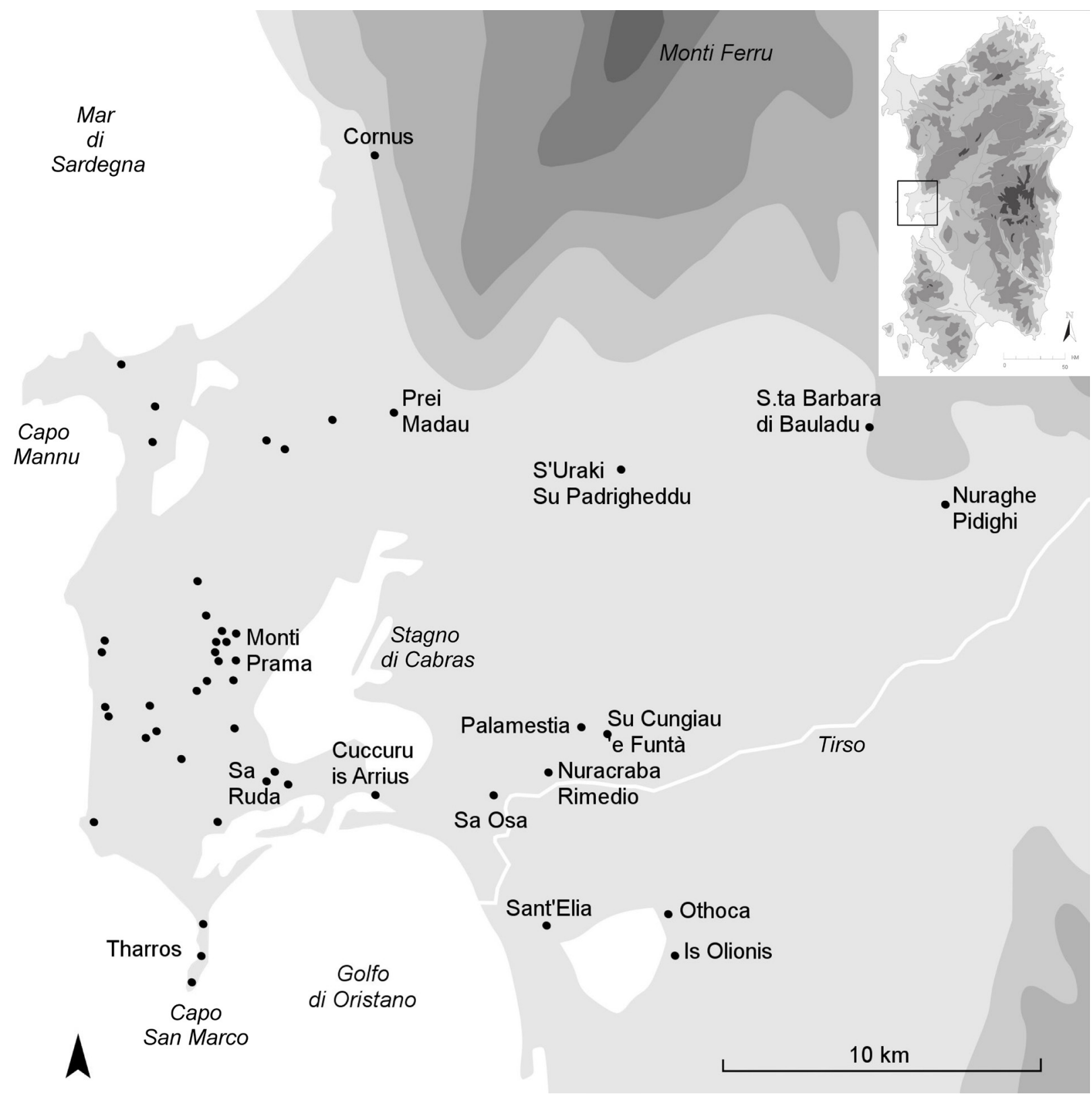

Figura 1. Localizzazione di S’Urachi/Su Padrigheddu e dei siti nuragici e fenici dell'età del Ferro nella Sardegna centro-occidentale. 


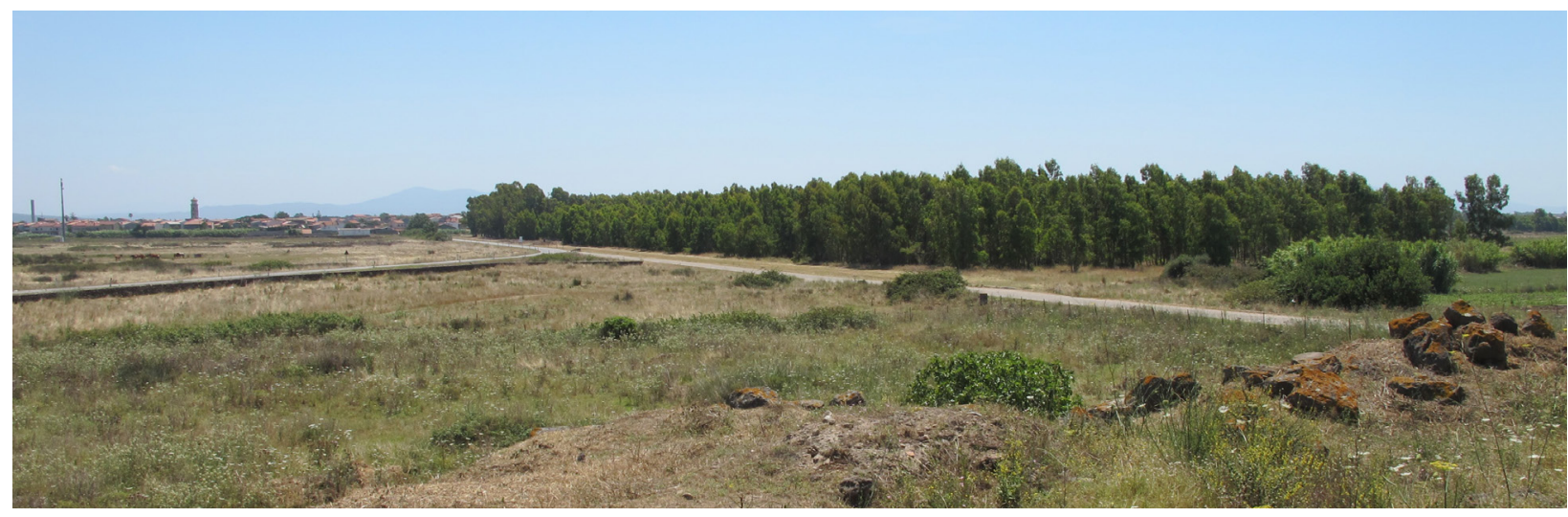

Figura 2. Vista da nuraghe S’Urachi del sito di Su Padrigheddu (il bosco di eucalipti) e del villaggio di San Vero Milis.

logiche di circolazione di questi materiali, cogliere le modalità attraverso le quali la comunità di S'Urachi e Su Padrigheddu intratteneva rapporti con i nuovi arrivati e definire le pratiche di uso di questi manufatti. Questo contributo è parte delle ricerche condotte nell'ambito del progetto Colonial Traditions, diretto dallo scrivente e da P. van Dommelen alle Università di Glasgow e Leicester fra 2009 e 2013. Tale progetto ha avuto come obiettivo lo studio delle pratiche ceramiche presso il sito di S'Urachi ed altri casi-studio sardi al fine di cogliere le interazioni fra popolazioni nuragiche e Fenici nel corso dell'età del Ferro, e i successivi sviluppi nel corso del periodo punico. Questo lavoro deriva anche dal coinvolgimento dello scrivente nelle indagini stratigrafiche condotte nel contesto del Progetto S'Urachi, iniziato nel 2013 sotto la direzione di P. van Dommelen e A. Stiglitz (http://blogs.brown. edu/surachi/)

\section{Le Ricerche a S'URAchi E a Su Padrigheddu}

La frequentazione di nuraghe S'Urachi abbraccia un arco temporale molto ampio, non solamente limitato alle fasi insediative e abitative comprese fra i secoli finali del II millennio a.C. e l'epoca romana, ma protratto anche in età post-antica in seguito all'abbandono in chiave residenziale del complesso. In questo senso, le vicende del nuraghe sono intimamente connesse a quelle del vicino villaggio di San Vero Milis, che ha restituito evidenze archeologiche di insediamento a partire dall'età romana imperiale. Le abitazioni del villaggio furono infatti edificate sia con i blocchi provenienti dal crollo del nuraghe, sia - e soprattutto - con mattoni crudi fabbricati con l'argilla estratta dal sito (figura 2).
La visibilità e la presenza del nuraghe sono documentate in modo precoce anche nella letteratura archeologica, sin dal 1935, quando l'allora Sovrintendente "agli scavi e musei archeologici della Sardegna” A. Taramelli ne menzionò l'esistenza nella sua Carta Archeologica (Taramelli, 1935, 110). Ricerche ufficiali e sistematiche al nuraghe presero avvio negli anni immediatamente successivi al secondo conflitto mondiale quando G. Lilliu operò una rapida campagna di scavi per conto della locale Soprintendenza (Lilliu, 1950). Quelle ricerche permisero di mettere in luce l'ingombro perimetrale del nuraghe e di disegnarne una prima planimetria (Contu, 1952, fig. 5), e di individuare delle strutture di epoca punica e repubblicana che si addossavano al perimetro esterno del monumento. Tuttavia, il metodo di indagine impiegato all'epoca, che prevedeva lo scavo di profonde trincee contigue al lato esterno dell'antemurale nuragico, unito al primario interesse dello scavatore verso le fasi nuragiche del monumento a scapito della comprensione del suo utilizzo in epoche successive, se da un lato permisero una rapida individuazione dell'ingombro del complesso - messo in luce nell'arco di pochi mesi -, d'altro lato ebbero come risultato la distruzione delle strutture delle fasi post-nuragiche. Nuraghe S'Urachi risultò formato da un antemurale di cui furono individuate sette delle almeno dieci torri che lo componevano. Non fu possibile mettere in luce il perimetro completo del complesso dal momento che la presenza di una contigua strada non rendeva fattibile la prosecuzione dei lavori (figura 3).

Dopo un periodo di stasi nelle ricerche fra gli anni Cinquanta e Settanta dello scorso secolo, periodo durante il quale furono apportati degli interventi di restauro al nuraghe, i lavori ripresero alla fine 
degli anni Settanta, sotto la spinta di G. Tore (Università di Cagliari). Le indagini furono questa volta concentrate all'interno del nuraghe, sulla sommità del monumento ed estese anche alla comprensione delle fasi post-nuragiche di vita del sito. Le ricerche stratigrafiche permisero di appurare che la stratificazione archeologica sulla sommità del complesso era composta da un potente strato di argilla e che si era formato in epoca post-antica a seguito di lavori di estrazione di argilla finalizzata alla produzione di mattoni crudi. Quegli scavi, protrattisi sino agli anni Novanta dello scorso secolo, non riuscirono a raggiungere livelli in giacitura primaria utili per una periodizzazione su basi stratigrafiche all'in- terno del nuraghe (Tore 1984). La cronologia del materiale recuperato, riferibile a tutte le fasi di vita del sito, se da un lato ha testimoniato inquivocabilmente il lungo periodo di insediamento antropico a S'Urachi (Roppa, Hayne e Madrigali 2013), d'altro lato ha posto la problematica delle attività a cui imputare la formazione di un potente strato di argilla sulla cima di un complesso - il nuraghe - di fatto realizzato con grosse pietre messe in opera a secco. Una possibile spiegazione può essere fornita dalla stessa cronologia dei materiali rinvenuti nel corso di quegli scavi, abbondantissimi e in larga parte databili alle fasi puniche e romana repubblicana, momento durante il quale la fisionomia stessa del

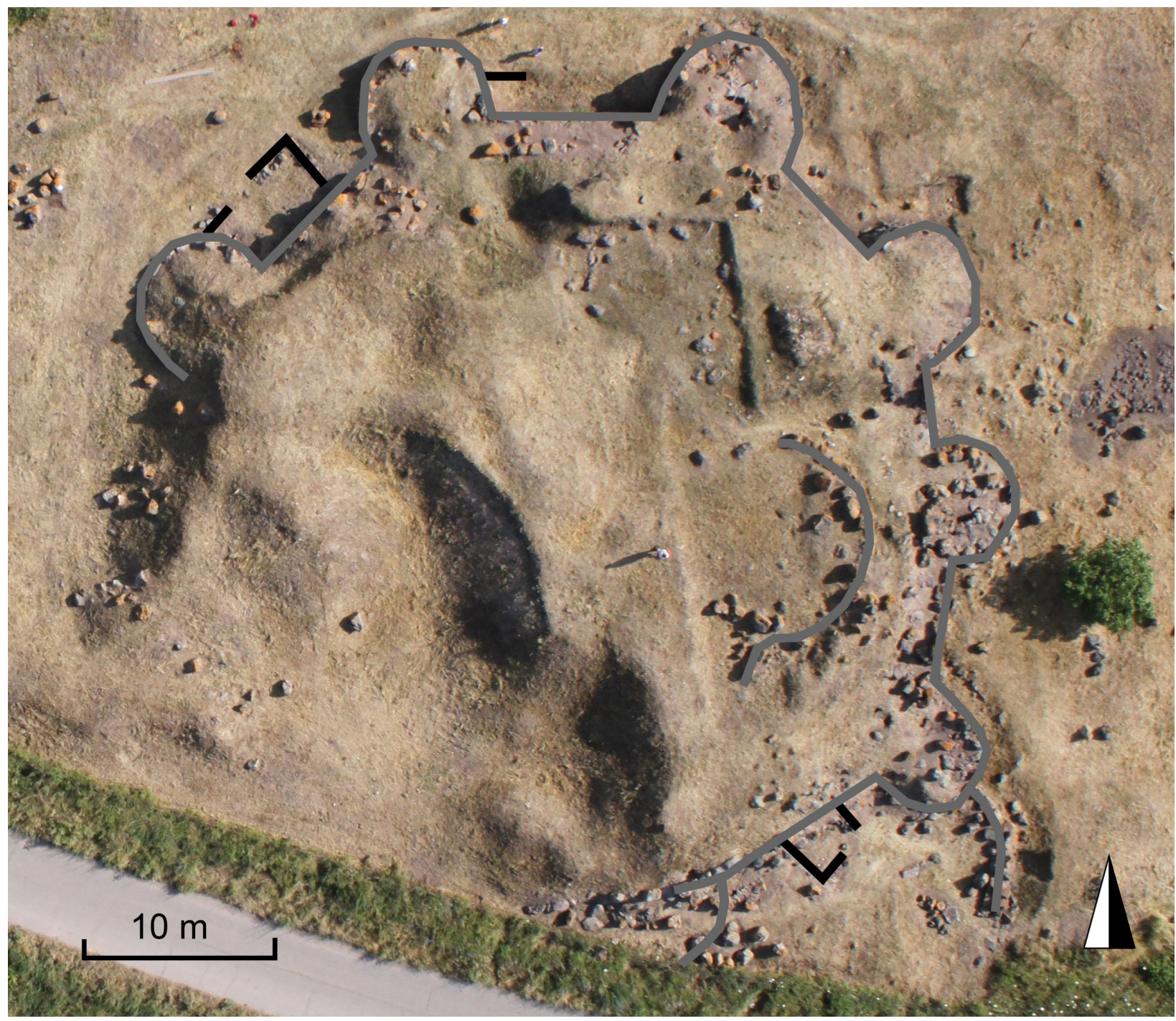

Figura 3. Foto aerea del nuraghe precedente agli scavi del 2013 con sovrapposizione della pianta delle strutture nuragiche (in grigio) e di epoca punica e romana repubblicana (in nero), come messe in luce dagli scavi Lilliu e Tore. Elaborato da Google Earth. 
monumento doveva essere stata fortemente mutata dalla costruzione di strutture in gran parte formate da mattoni crudi all'esterno e al di sopra dei crolli del nuraghe. Il successivo difacimento dell'argilla dei mattoni, dovuto all'azione meteorica, può presumibilmente essere indicato come una delle cause della formazione del potente deposito terragno sulla sommità del complesso.

Nel corso degli stessi anni in cui si svolgevano le ricerche stratigrafiche nel nuraghe, dei lavori agricoli per la piantumazione di un boschetto di eucalipti in un appezzamento in stretta contiguità con il complesso nuragico denominato Su Padrigheddu permisero di recuperare un cospicuo lotto di materiali ceramici. Il proseguio dei lavori agricoli impedì purtroppo un'indagine stratigrafica approfondita e la folta vegetazione nel frattempo cresciuta rende oggi minima la visibilità archeologica -, ma la cronologia e la varietà funzionale del materiale recuperato hanno reso possibile di identificare nel sito un villaggio attivo lungo tutto il I millennio a.C., contemporaneo quindi alle fasi di vita documentate a S’Urachi (Tore, 1984; Stiglitz, 2007; Roppa, 2012).

In anni più recenti, dal 2013, un progetto di indagine archeologica ad ampio raggio - Progetto S'Urachi - è attivo sul sito, diretto da P. van Dommelen (Brown University) e A. Stiglitz (Comune di San Vero Milis), che mira a studiare il complesso nuragico come un monumento di età storica, classica e romana, esaminandone il significato per le comunità locali dall'età del Ferro sino ad epoca romana. Le indagini, seppur ad uno stadio ancor embrionale e concentrate all'esterno del nuraghe, hanno permesso di individuare nuove strutture a pianta quadrangolare di epoca punica e romana repubblicana addossate al perimetro esterno dell'antemurale e, in alcuni casi, costruite al di sopra dei crolli dell'imponente complesso nuragico, testimoniando così una rifunzionalizzazione del monumento preistorico.

\footnotetext{
S'URAchi/Su Padrigheddu Nel CONTESTO RegioNALE

Nuraghe S’Urachi e l'adiacente sito di Su Padrigheddu sono collocati nella piana del Campidano settentrionale, nella pianura alluvionale del fiume Tirso, a nord del golfo di Oristano (fig. 1). A ovest, separato dalla dorsale collinare della penisola del Sinis e da una serie di stagni si trova la costa occidentale dell'isola, esposta al mar di Sardegna. Il monumentale nuraghe rappresenta il centro più importante di tutta l'area, come testimoniato dal-
}

la protratta continuità di vita fra età del Bronzo ed epoca romana repubblicana. Il settore a nord del golfo appare interessato da vivaci dinamiche sociali nel corso dell'età del Ferro (IX-VI sec. a.C.), che coincide a partire dall'VIII sec. a.C. con un forte incremento nella circolazione di materiali di tipologia fenicia e lo stanziamento nel corso del VII sec. a.C. di gruppi umani di tradizioni culturali fenicie. Questa aumentata mobilità di beni e di persone sembra apparentemente accompagnata da vistosi cambiamenti nel corpo sociale delle locali comunità nuragiche, che secondo la tradizionale ricostruzione storico-archeologica conobbero nel corso dell'età del Ferro una sostanziale contrazione insediativa. Tuttavia, una più approfondita seriazione della ceramica nuragica dell'età del Ferro e recenti ricerche di superficie hanno permesso di cogliere in molti siti sostanziali continuità nel corso del periodo in questione, continuità che appare in alcuni casi - in primis a S'Urachi/Su Padrigheddu - anche testimoniata dalla presenza di materiale fenicio, generalmente meglio datato della ceramica nuragica (per un quadro su queste problematiche: Roppa, 2012, 5-8 con bibliografia).

Particolarmente significativo per la comprensione dei cambiamenti in seno alle locali comunità nuragiche e per il ruolo che dovette aver ricoperto S’Urachi nelle dinamiche regionali del periodo è il sito di Monti Prama, localizzato su una bassa altura lungo la dorsale del Sinis a sudovest rispetto a S'Urachi/Su Padrigheddu. L'area è molto nota per la sua valenza cimiteriale dal IX sec. a.C. e soprattutto per l'eccezionale complesso scultoreo che fu qui rinvenuto. La fase più significativa del complesso è databile a partire dalll'VIII sec. a.C., periodo a partire dal quale si data un allineamento di trentatré inumazioni in pozzetto, tutte prive di corredo tranne una delle deposizioni più tarde (n. 25) che ha restituito un sigillo scaraboide (Tronchetti e van Dommelen 2005; Stiglitz 2007). A una fase successiva, probabilmente da porre nel VII sec. a.C. data un'ulteriore serie di inumazioni - note come scavi Bedini -, parzialmente a cista litica, direttamente a nord dell'allineamento di sepolture in pozzetto, e da questo separato mediante un asse viario (figura 4).

Una sostanziale differenza fra le diverse fasi della necropoli è l'associazione del gruppo di sepolture in pozzetto con un cospicuo gruppo scultoreo, rinvenuto frammentario in giacitura secondaria in uno strato che fu direttamente sovrapposto alle 


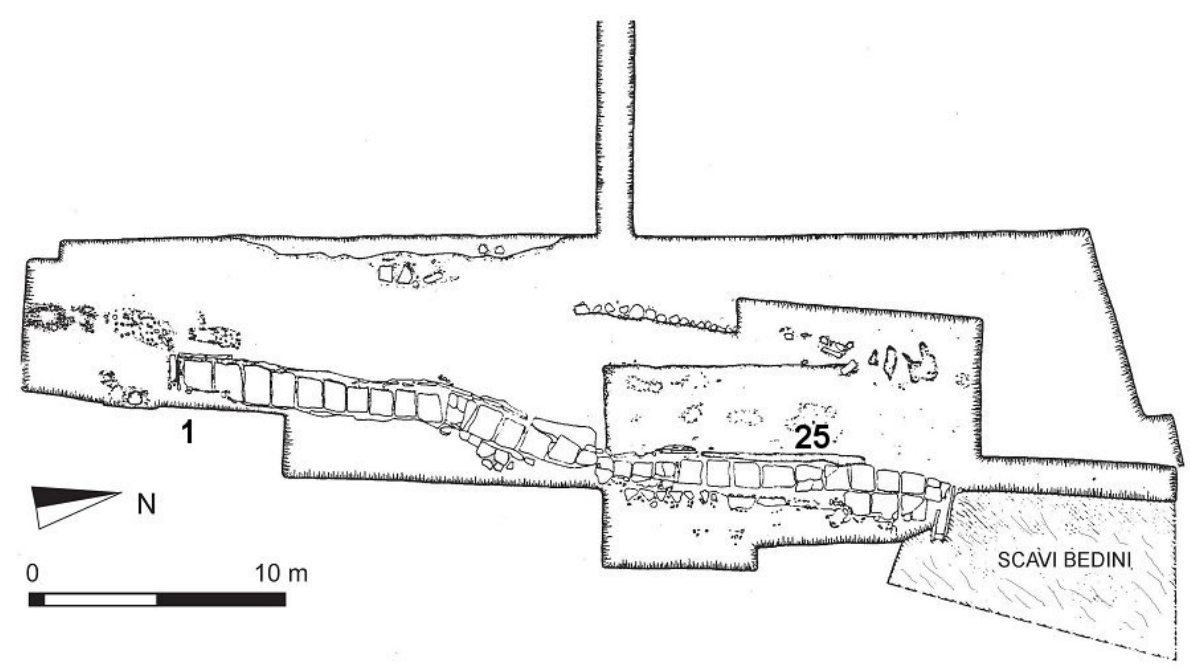

Figura 4. Planimetria delle sepolture dell'età del Ferro di Monti Prama. La sepoltura più antica è la numero 1, a sud. Elaborato da Tronchetti, van Dommelen 2005, figura 3.

tombe non prima della fine del IV sec. a.C. In questo accumulo, furono rinvenuti più di cinquemila frammenti scultorei, che i restauri tuttora in corso hanno reso possibile rapportare ad almeno venticinque statue di figure umane, ventotto modelli di nuraghe e sette betili. Fra le statue, tutte di altezza superiore ai due metri, sono stati identificati diciassette pugilatori, sei arcieri e due guerrieri (Bedini, Tronchetti, Ugas e Zucca 2012). Mentre l'interesse degli studiosi si è immediatamente focalizzato sull'eccezionalità del gruppo scultoreo rinvenuto, il contesto complessivo del sito è stato spesso trascurato. La necropoli si trova infatti in un'area a densissimo popolamento nuragico, dato che in un raggio di poco più di $1 \mathrm{~km}$ si trovano cinque nuraghi (Molas, Sa Tiria, Cannevadosu, Su Archeddu su Procou, Monti Prama) due dei quali circondati da altrettanti villaggi, mentre a poca distanza dalla stessa area cimiteriale si trova un terzo villaggio. Da un punto di vista cronologico, materiali della prima età del Ferro sono stati raccolti presso il nuraghe Cannevadosu e il villaggio di Monti Prama, mentre non sono disponibili dati cronologici per l'inquadramento dei restanti siti (Sebis, 1987; Sebis, 1998).

Nonostante la carenza di una precisa scansione cronologica del contesto areale di Monti Prama, appare evidente il radicamento e la continuità di forme di popolamento tradizionali per lo meno alla fine dell'età del Bronzo. Inoltre, se la prima fase della necropoli potrebbe essere messa in relazione con le comunità insediate negli insediamenti circostanti, l'eccezionale monumentalizzazione dell'area tra fine VIII e VII sec. a.C. corrisponde
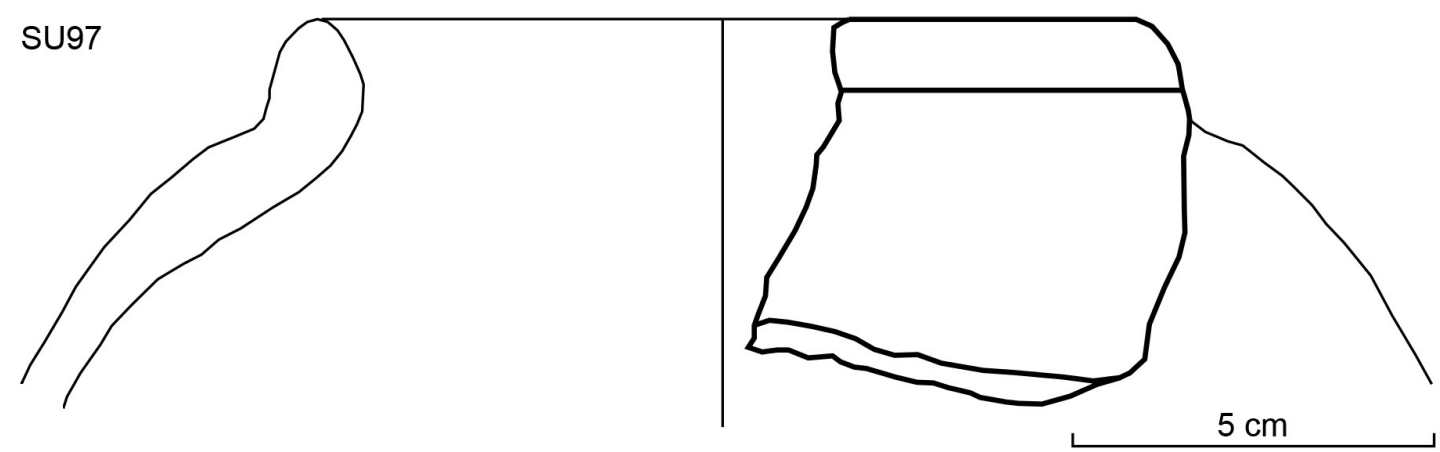

Fig. 5. Disegno del frammento SU97. 
a un chiaro rapporto fra statue e sculture in una sorta di heroon, in cui preminente appare la funzione auto-rappresentativa e simbolica veicolata dall'intero contesto rituale che si configura, a livello regionale, come l'espressione comunitaria del gruppo più influente del Sinis - alto Oristanese nel corso dell'età del Ferro (Lilliu, 2002; Tronchetti e van Dommelen, 2005; Stiglitz, 2007; Bedini, Tronchetti, Ugas e Zucca 2012). Dal momento che l'insediamento di primo piano dell'intera area è rappresentato da nuraghe S'Urachi, non sfuggono le connessioni fra i due siti ed è possibile ipotizzare una monumentalizzazione di Monti Prama fra VIII e VII sec. a.C. in relazione alla sua collocazione lungo il percorso da S'Urachi/Su Padrigheddu a Tharros e all'emergente ruolo del centro fenicio nelle dinamiche regionali.

A questa vitalità delle comunità nuragiche dell'età del Ferro si aggiunge nel corso del VII sec. a.C. il graduale stanziamento di gruppi extra-isolani di cultura fenicia nei centri di Tharros, localizzato lungo la stretta penisola di capo San Marco che chiude a nord il golfo di Oristano, ed Othoca, sulla sponda settentrionale del golfo. La datazione delle fasi iniziali dell'insediamento a Tharros si colloca lungo il VII sec. a.C., grazie alle evidenze fornite dal tofet e dalle necropoli ad incinerazione (Bernardini, 2005), mentre è collocato tra le fasi finali del VII sec. a.C. e gli inizi del secolo successivo l'insediamento fenicio di Othoca, supportato dalla cronologia dei materiali della necropoli ad incinerazione e dalle stratigrafie della modesta porzione di abitato posta in luce (Nieddu e Zucca, 1991, 48, 120). Particolarmente rilevante è in entrambi i siti la preesistenza di siti nuragici, sin dall'età del Bronzo a Tharros (Bernardini, 1997), dalla prima età del Ferro a Othoca (Atzori, 1992; Nieddu e Zucca, 1991, 48). Significativa è la presenza nel sito di capo San Marco di materiale di possibile importazione micenea della tarda età del Bronzo, che testimonierebbe quindi la circolazione di materiale extra-isolano nell'area a nord del golfo di Oristano sin da momenti precoci (Bernardini, 2005).

A livello regionale, il contesto entro il quale sono inseriti i siti di S’Urachi/Su Padrigheddu appare quindi denotato da una forte vivacità nel corso dell'età del Ferro e in coincidenza dell'incrementata mobilità di materiali e persone a partire dall'VIII sec. a.C.
I MATERIALI FENICI DA S'URACHI/SU PADRIGHEDDU ${ }^{1}$

I materiali dai siti esaminati sono stati oggetto di approfondite analisi nell'ambito del progetto Colonial Traditions, che ha in particolare posto l'attenzione sulle pratiche di produzione ceramica e su aspetti artigianali e tecnici della produzione. In questa sede, mi propongo di esaminare i materiali fenici provenienti dai recuperi di emergenza presso Su Padrigheddu e dagli scavi degli anni Ottanta presso il nuraghe S'Urachi. Questi vengono analizzati non solo per comprendere i traffici commerciali entro cui l'insediamento era inserita, ma anche come evidenza della graduale assunzione di manufatti non tradizionali in seno alla comunità locale e, possibilmente, di nuove pratiche di uso e modi di consumo che potrebbero essere da questi testimoniati (Hayne, Madrigali e Roppa c.s.).

Nello specifico, tutti i reperti raccolti in occasione dei recupero di emergenza presso Su Padrigheddu sono stati esaminati, mentre solo una minima parte dei materiali rinvenuti nel corso degli scavi a S’Urachi è stata analizzata. Fra i quasi 111000 reperti visionati, che corrispondono a meno della metà dei materiali portati complessivamente alla luce nel corso degli scavi degli anni Otttanta dello scorso secolo, molto ridotto è il numero di materiali dell'età del Ferro, tra i quali quelli di tradizione fenicia, selezionati su criteri tipologici e presentati nel presente lavoro. Di contro, fra i materiali provenienti da $\mathrm{Su}$ Padrigheddu sono molto più abbondanti i reperti dell'età del Ferro. Questo può essere imputato alla natura dei due depositi, meno profonda e non interessata a rimaneggiamenti successivi a Su Padrigheddu, cosicché gli scassi profondi effettuati per la piantumazione degli eucalipti intaccarono gran parte della stratificazione archeologica. Diverse e più complesse sono invece la situazione deposizionale e le vicende post-deposizionali presso il nuraghe, dove ad una probabile fase edilizia molto significativa connotata dal massiccio impiego di mattoni crudi al di sopra dei crolli e delle strutture del nuraghe, nella seconda parte del I millennio a.C., seguì una lunga fase durante la quale il sito funse da cava di argilla e materiale lapideo. Per questo motivo, può essere che i materiali più antichi, del periodo trattato in questa sede, siano stati sigillati al di sotto delle fasi strutturali di epoca punica e romana repubblicana.

1 I prefissi SU e SP nei numeri identificativi dei reperti si riferiscono rispettivamente alla provenienza dei materiali da S'Urachi e Su Padrigheddu rispettivamente. 

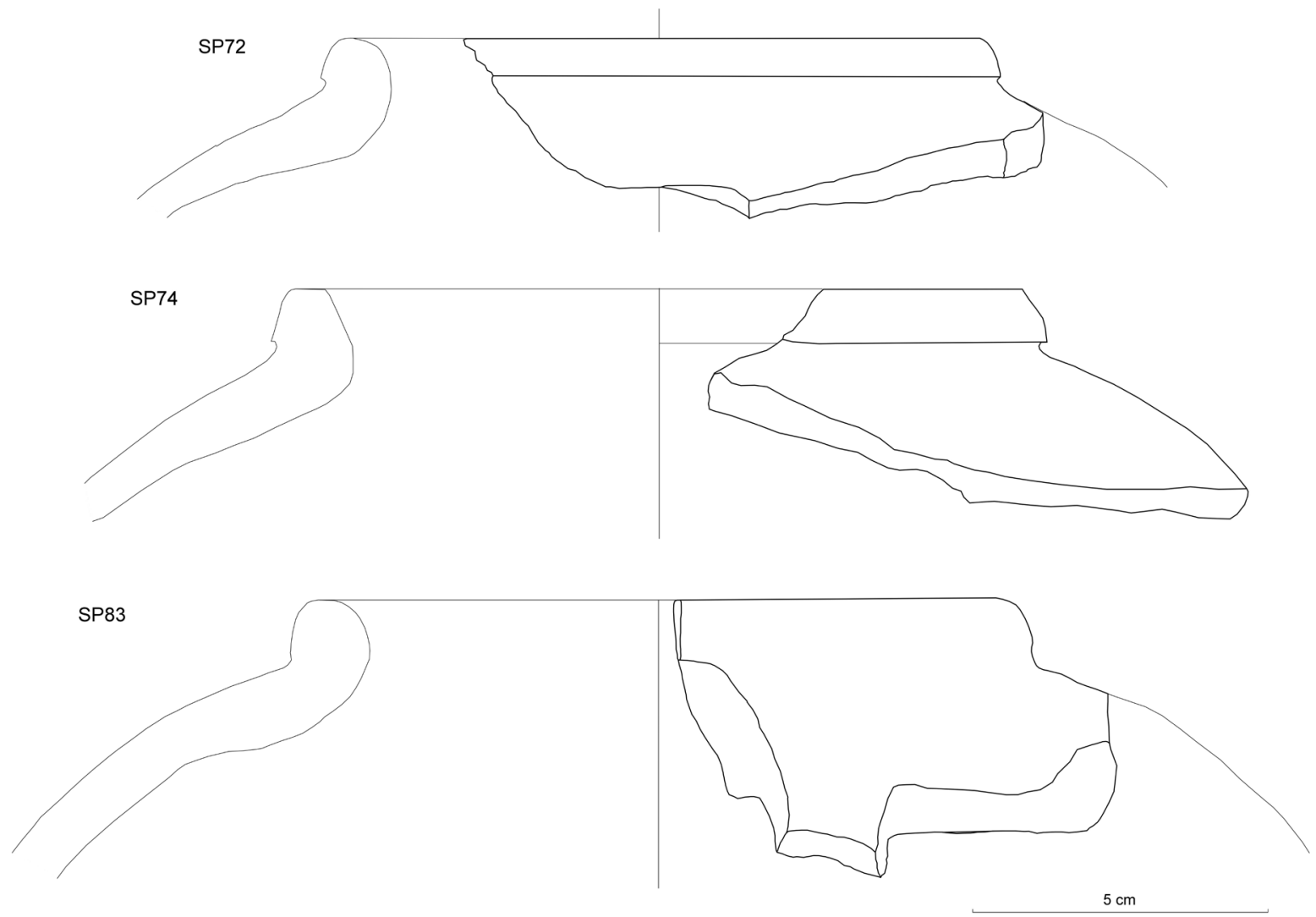

Figura 6. Disegni di frammenti rappresentativi della tipologia anforica Ramón T-2.1.1.2. = Bartoloni B7.

Nel presente contributo vengono esaminati manufatti ceramici rispettivamente relativi alle classi funzionali delle anfore, della ceramica da cucina/ preparazione e della ceramica da mensa. Nonostante l'attenzione venga qui posta in particolare su aspetti tipologici, gli impasti - fabrics - di tutti i materiali sono stati esaminati sia da un punto di vista macroscopico e quantitativo, che microscopico e qualitativo mediante analisi petrografiche di sezioni sottili. Mentre si rimanda ad altri contributi per l'analisi e discussione dettagliata dei fabrics e degli aspetti tecnici e artigianali del processo di produzione delle ceramiche da S’Urachi e Su Padrigheddu (Roppa, 2012; Roppa, Hayne e Madrigali, 2013; Roppa, 2014), in questa sede si rimarca che due gruppi di fabrics (SU1 e SU2 in Roppa $2012=$ SVM1 e SVM2 in Roppa, Hayne e Madrigali 2013) sono stati identificati come produzioni locali, e che in entrambi i gruppi è presente ceramica di tipologia fenicia. In particolare, il gruppo 1 denota la tradizione ceramica locale nuragica che affonda le sue radici nell'età del Bronzo, mentre il gruppo 2 risulta esclusivamente utilizzato per la manifattura di ceramica di tipologia fenicia a partire dalla seconda metà del VII sec. a.C.

\section{Le anfore}

Tra i materiali esaminati, i frammenti di anfora costituiscono certamente la classe funzionale maggiormente rappresentata per il periodo in questione. Si tratta di cinquantacinque frammenti di orli, distribuiti in modo disomogeneo fra la seconda metà dell'VIII sec. a.C. e la fine del VI sec. a.C. Il reperto più antico è l'orlo SU97 (figura 5), avvicinabile alla tipologia T-3.1.1.1. di J. Ramón Torres (Ramón Torres, 1995), corrispondente al modello B1 nella seriazione tipologica isolana elaborata da P. Bartoloni (Bartoloni, 1988). Nello specifico, il reperto appare accostabile alla cosiddetta "variante sulcitana", un modello recentemente isolato da M. Botto, caratterizzato da "orlo, dritto o leggermente inclinato verso l'interno, che risulta schiacciato internamente, talvolta con leggera sagomatura esterna", la cui area di produzione sembra essere compatibile sulla base di analisi archeometriche con il distretto regionale del Sulcis e nello specifico con 
l'abitato di Sant'Antioco, nell'estremo meridione della Sardegna, uno dei settori isolani di più precoce interesse da parte dei Fenici (Botto, Deriu, Negri, Oddone, Segnan e Trojsi, 2005, 69). Tale modello è stato anche individuato fra i materiali recuperati nel corso degli scavi presso il foro di Nora, presso la costa sudorientale dell'isola, dove la recente edizione dello scavo ha messo bene in luce la presenza di questa interessante e precoce variante tipologica di produzione isolana (Finocchi, 2009, 379-385). In particolare, l'esemplare da S'Urachi presenta delle similarità con un esemplare dal sito del Cronicario presso Sant'Antioco (Botto, Deriu, Negri, Oddone, Segnan e Trojsi, 2005, 66, fig. 1, g). Sulla base di confronti con i materiali provenienti dai contesti stratigrafici ben datati di Sant'Antioco, la datazione di questa tipologia si pone fra la metà dell'VIII e gli inizi del VII sec. a.C. L'esemplare qui presentato appare accostabile a questa tipologia anche sulla base delle caratteristiche dell'impasto, che mostra sostanziali analogie con i reperti anforici identificati in questa variante provenienti dagli scavi di Nora (Finocchi, 2009, 388, n. 28-30).

Un maggiore afflusso di anfore fenicie nel sito appare testimoniato tra la fine del VII e gli inizi del VI sec. a.C. dalla tipologia Ramón T-2.1.1.2. = Bartoloni B7, rappresentata a S’Urachi e Su Padrigheddu da quattordici esemplari. Questa tipologia, caratterizzata da un orlo a frequente sezione triangolare separato mediante un solco o un'incisione dalla spalla con forte andamento convesso, rappresenta "l'ultimo anello formale apparentabile ai prototipi occidentali e il primo indizio di mutazione locale che ... si manifesta in modo evidente a partire dalla fine del VII sec. a.C.” (Bartoloni, 1988, 38). Di particolare importanza appare la produzione locale isolana di questo modello anforico, che è attestata come prima produzione anforica anche presso altri siti fenici sardi - ad esempio Nora (Finocchi, 2009, 384) -, e che appare testimoniata anche nei materiali qui presentati. Dei quattordici orli pertinenti a questa tipologia, uno sembra presentare caratteristiche compatibili con una produzione locale (SP60) (Roppa, 2012, 15), nove mostrano similarità con i materiali di probabile produzione coloniale di Tharros (SU72, 75, 76, 79, SP16, 94, 42, 57, 67, 134) (van Dommelen e Trapichler, 2011; Roppa 2012, 18-19; Roppa, Hayne e Madrigali, 2013, 127), mentre i restanti quattro frammenti (SU68, 81, 126, SP66) presentano caratteristiche non associabili con i fabrics individuati dalle ricerche (fig. 6)

Cronologicamente poco successivi sono i dieci frammenti di anfora modello Ramón T-1.2.1.1. = Bartoloni D1, collocabile nella prima metà del VI sec. a.C. Fra questi, l'esemplare SP70 presenta delle similarità morfologiche anche con la tipologia Ramón T-1.4.2.1. = Bartoloni D2-3, di datazione successiva a coprire la seconda metà del VI sec. a.C., che ne suggeriscono quindi una generica collocazione temporale lungo tutto il VI sec. a.C. La produzione degli esemplari esaminati appare in due casi riferibile ad un contesto locale (SP61, 62) (Roppa, 2012, 15), in quattro casi probabilmente associabile con una produzione tharrense (SP76, 80, $84,85)$, e di incerta provenienza per i rimanenti quattro esemplari (SP70, 89, 91, 92) (fig. 7).

La tipologia anforica presente in modo maggioritario è però costituita dal più tardo tipo Ramón T-1.4.2.1. = Bartoloni D2-3, rappresentato a S'Urachi e a Su Padrigheddu da trenta frammenti. Tale gruppo tipologico, che deriva come il precedente dal modello Ramón T-2.1.1.2. = Bartoloni B7, è caratterizzato da un corpo a sacco e da un orlo gonfio e impostato direttamente sulla spalla, che acquisisce un andamento maggiormente obliquo. La presenza di questa tipologia è molto diffusa in tutta l'isola

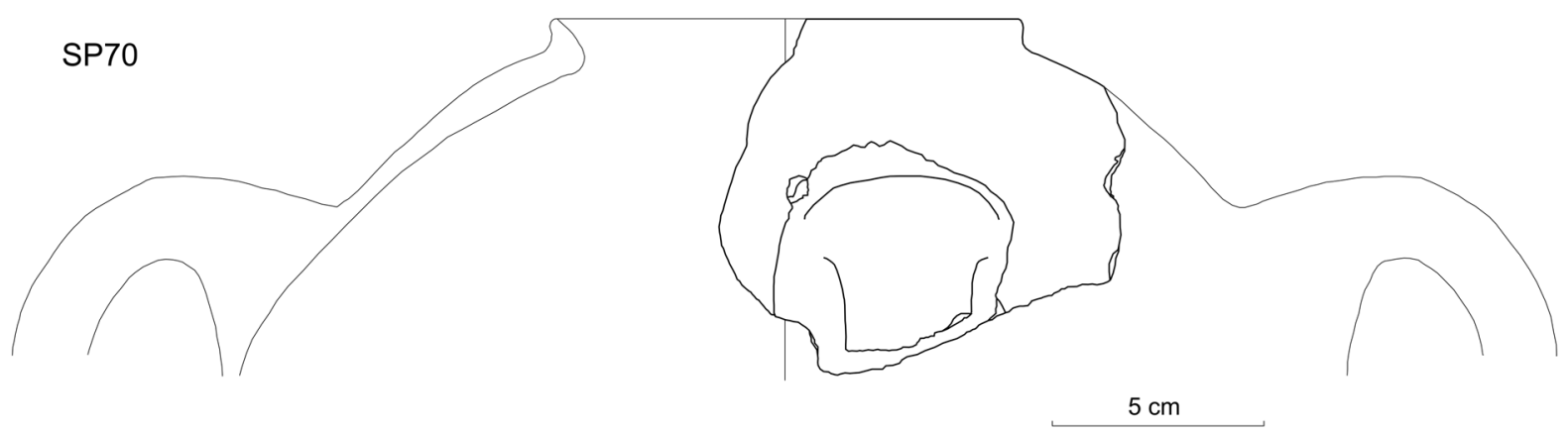

Figura 7. Disegno del frammento SP70. 
lungo tutto il VI sec. a.C. sino a raggiungere in alcuni casi gli inizi del V sec. a.C. (Finocchi, 2009, 402403). Tra gli esemplari esaminati, tre (SU46, SP65, 90) presentano dei fabrics compatibili con una produzione locale, tredici (SU85, 91, 101, SP72, 74, $77,78,81,82,83,86,87,98)$ mostrano stringenti similarità con fabrics di probabile produzione tharrense, mentre di difficile inquadramento sono i restanti quattordici frammenti (SU32, 35, 64, 74, 83, 90, 92, SP63, 88, 92, 93, 94, 96, 102) (fig. 8).

\section{La ceramica da cucina e preparazione}

A questo insieme funzionale sono state ricondotte sostanzialmente due forme ceramiche, forme chiuse con primario utilizzo per la cottura dei cibi - cooking pots - e forme aperte funzionali alla preparazione e miscelazione di alimenti - bacini.

La prima forma ceramica è testimoniata a S'Urachi e a Su Padrigheddu da quindici esemplari, riconducibili a diverse varianti tipologiche che si collocano cronologicamente fra VII e VI sec. a.C., con alcune forme che sono stratigraficamente attestate anche in contesti isolani di V sec. a.C. Per quanto concerne i fabrics, tutti i materiali esaminati presentano delle caratteristiche che li rendono compatibili con una produzione locale: in particolare due frammenti di orlo $(\mathrm{SP} 48,49)$ possono essere raggruppati nell'insieme 1 che caratterizza i materiali di tradizione nuragica, mentre i restanti esemplari sono raggruppabili nel gruppo 2 , fabric che sulla base di criteri tipo-cronologici fece la sua comparsa nel sito probabilmente a partire dalla metà del VII sec. a.C.

Facendo riferimento alla seriazione tipologica proposta da L. Campanella per i materiali provenienti dagli scavi di Nora (Campanella, 2009a), tutti i materiali sono stati ricondotti nel macrogruppo tipologico P2, che presenta al suo interno delle talvolta minime variazioni formali. Tra gli esemplari più antichi si segnalano due esemplari (SP47, 49) pertinenti alla tipologia $\mathrm{P} 2 \mathrm{~A}$, che identifica pentole monoansate di forma ovoide con orlo a pareti parallele estroflesso e bordo arrotondato, la cui datazione appare compresa nella seconda metà del VII sec. a.C. (Campanella, 2009a, 300-301). Quattro frammenti (SP41, 48, 51, 52) appaiono invece accostabili alla tipologia $\mathrm{P} 2 \mathrm{~B}$, simile alla precedente ma con un ispessimento e un appiattimento del bordo, la cui cronologia oscilla fra la fine del VII sec. a.C. e la metà del secolo successivo (Campanella, 2009a, 300). Fra questi, l'esemplare SP52 è del tipo con "falso versatoio", ed è confrontabile rispettivamente con esemplari dagli scavi del tofet di Sant'Antioco (Montis, 2004, 88, tipo CT.PG, n. 3, 6) e dalla stessa Nora (Campanella, 2009a, 305, n. 25) (fig. 9).

Due frammenti $(\mathrm{SP} 40,42)$ sono pertinenti alla
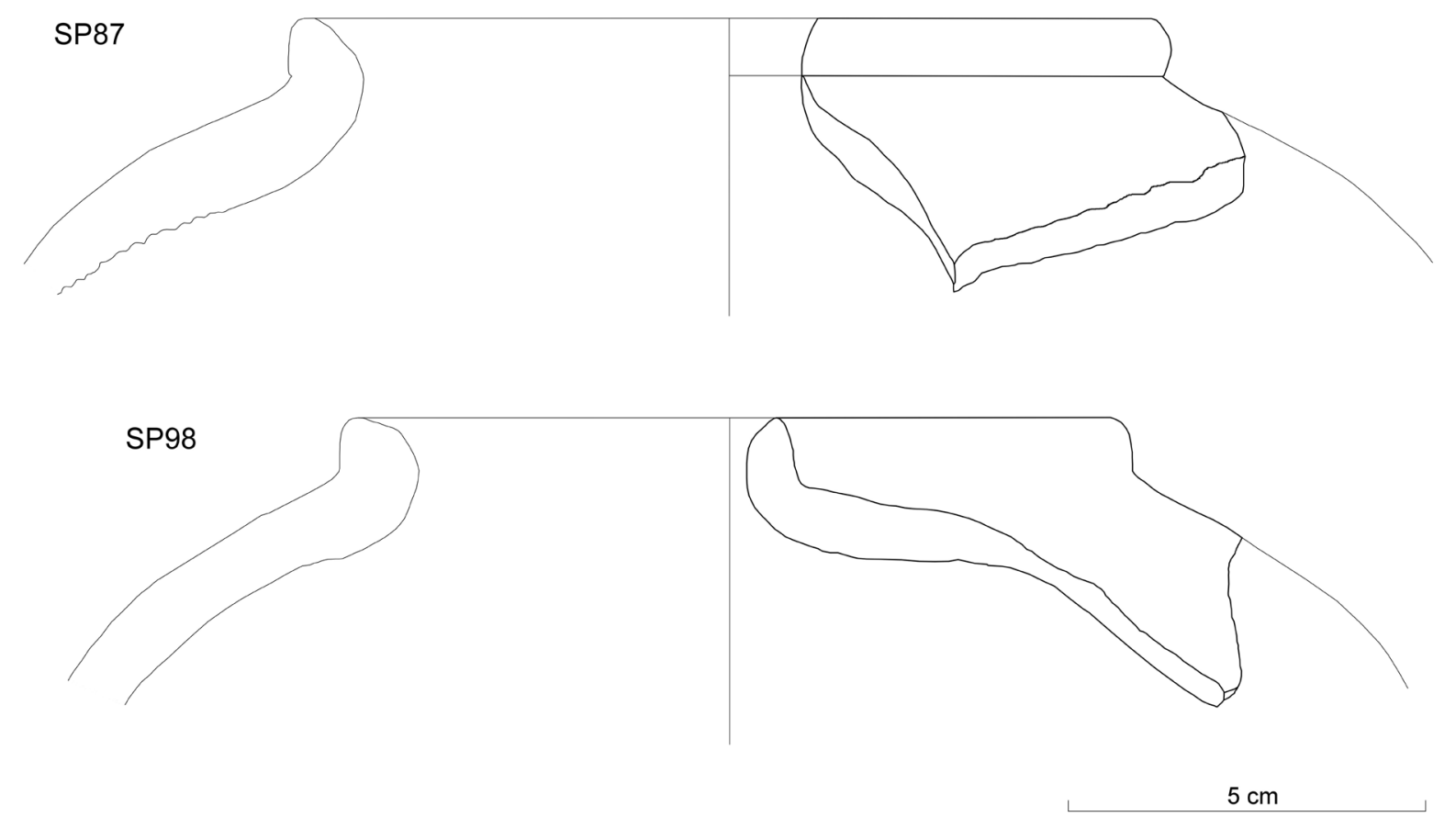

Figura 8. Disegno di frammenti rappresentativi della tipologia anforica Ramón T-1-4.2.1. = Bartoloni D2-3. 


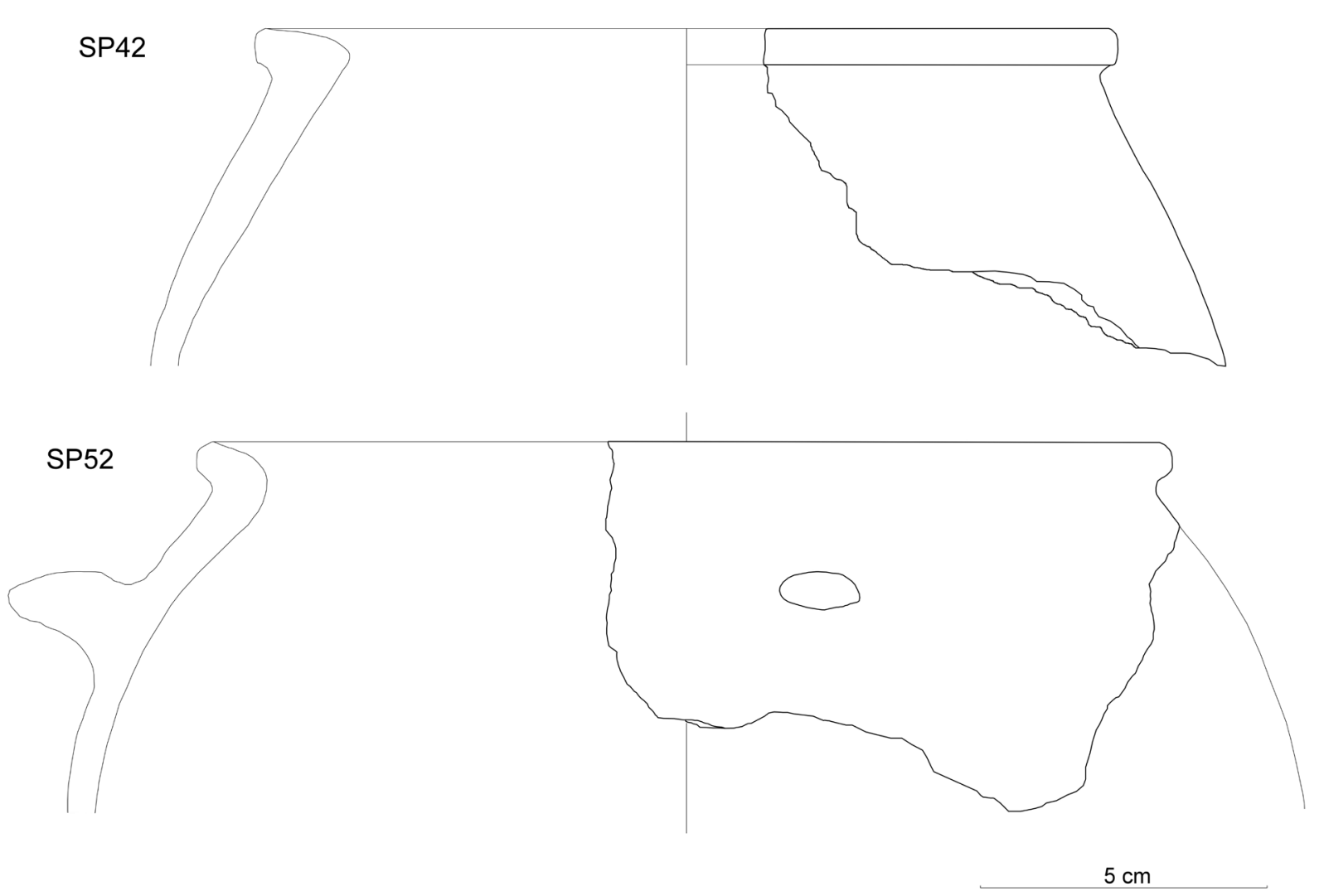

Figura 9. Disegni di frammenti di cooking pots.

tipologia P2C, databile nella seconda metà del VI sec. a.C., e quattro esemplari $(S P 45,54,56,57)$ appaiono tipologicamente collocabili nel gruppo P2D, caratterizzata da un orlo a collaretto, la cui cronologia è compresa fra i decenni conclusivi del VI sec. a.C. e il secolo successivo, dunque in età pienamente punica (Campanella, 2009a, 300). Tre frammenti (SP43, 44, 50), a causa delle cattive condizioni di conservazione, non risultano identificabili (fig. 10).

Per quanto concerne i materiali ceramici pertinenti alla forma dei bacini, tredici esemplari sono stati individuati e sono stati classificati tipologicamente sulla base della seriazione proposta per i materiali di Nora (Campanella, 2009b). Da un punto di vista dei fabrics, sei frammenti (SU118, 121, SP35, 37, 112, 113) sono identificabili nel gruppo 1 di produzione e tradizione locale, tre (SP38, 39, 69) appaiono raggruppabili nell'insieme 2 di produzione locale, ed altri tre (SP33, SU66, 117) non appaiono accostabili a nessuno degli insiemi di fabrics individuati.

Da un punto di vista tipologico, quattro esemplari (SP33, 39, 112, 113) sono riconducibili alla tipologia BA1, che include i bacini con orlo triangolare e vasca profonda, di datazione compresa fra il VII sec. a.C. e la prima metà del VI sec. a.C. (Campanella, 2009b, 248-249). Alla tipologia BA2, sempre caratterizzata da orlo triangolare ma con vasca bassa e di ampia copertura cronologica fra la seconda metà dell'VIII sec. a.C. e il VI sec. a.C. (Campanella, 2009b, 253), sono accostabili i due frammenti SP37 e 38, il primo dei quali può essere confrontato con un esemplare dalla necropoli di San Giorgio di Portoscuso, nell'estremo meridione dell'isola, in un contesto datato fra la metà dell'VIII e gli inizi del VII sec. a.C. (Bernardini, 2000, 39, fig. 6.3). Considerata comunque l'ampia copertura cronologica di questa tipologia, e la generale datazione più recente sia degli altri bacini che di tutti gli altri materiali contestuali, così come della ceramica fenicia prodotta nel fabric 1, è probabile che per questo esemplare sia da escludere una datazione così alta.

Di cronologia compresa fra la metà del VII sec. a.C. e il VI sec. a.C. è la tipologia BA3, distinta da un orlo a mandorla (Campanella, 2009b, 256257), e rappresentata fra i materiali di S'Urachi e Su Padrigheddu da quattro frammenti (SU117, 118, 121, SP36). Due frammenti (SU66, SP69) appaiono 


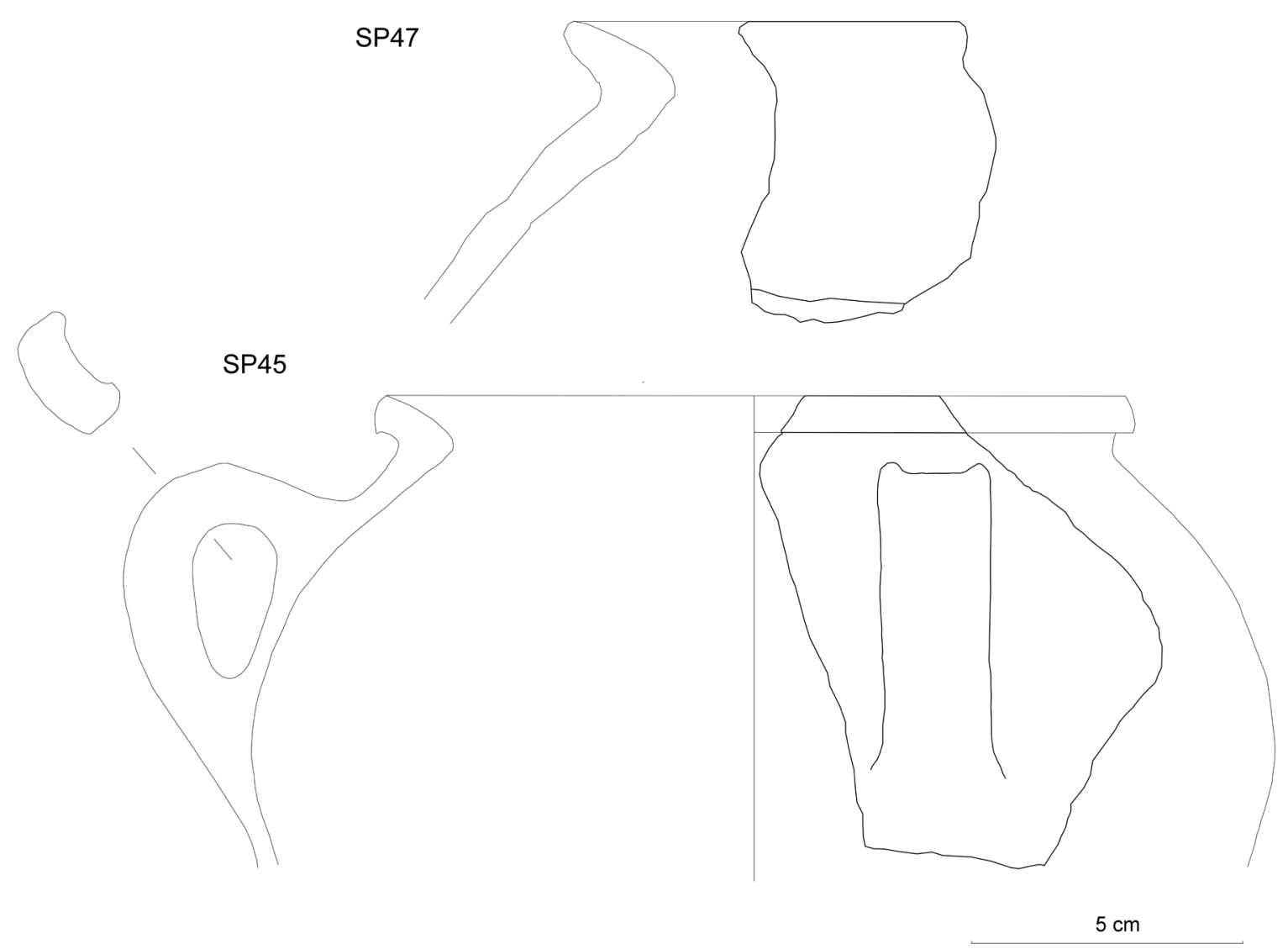

Figura 10. Disegni di frammenti di cooking pots.

collocabili nell'insieme BA5, la cui caratteristica distintiva è l'orlo ingrossato e pendulo e la cui datazione si colloca fra VII e VI sec. a.C. (Campanella, 2009, 263). A concludere la gamma tipologica dei bacini di S’Urachi e Su Padrigheddu è il modello BA6, con orlo ingrossato e piegato verso l'interno e di cronologia simile al tipo precedente, rappresentato dal frammento SP35 (figura 11)

\section{La ceramica da mensa}

Al gruppo funzionale della ceramica da tavola vanno ricondotte una serie di forme, tra cui dieci frammenti attribuibili ad altrettante coppe, quattro orli di piatti, un frammento di attingitoio - dipper -, ed un orlo di brocca, di cronologia variabile tra la metà dell'VIII sec. a.C. e il VI sec. a.C. (figura 12).

Fra le coppe, una delle tipologie maggiormente rappresentate con quattro esemplari (SU78, 102, 116, SP114), è la "coppa aperta con orlo ripiegato e solcato", in alcuni casi con rivestimento interno in red slip (SU102, 116, SP114), tipo che trova ampi riscontri nel Mediterraneo centro-occidentale, in particolare sull'isola con gli esemplari rinvenuti a Nora e datati fra il tardo VII e il VI sec. a.C. (Botto, 2009, 130-131). L'analisi dei fabrics di questi esemplari ha evidenziato compatibilità con una produzione locale per un frammento (SU78), la cui qualità è di molto inferiore rispetto ai restanti tre esemplari. Tra questi, di probabile provenienza dal bacino tharrense sono i due frammenti SU102 e 116, mentre di incerta produzione è il frammento SP114. Rappresentata da tre esemplari (SU21, 63, 70) è la tipologia della "coppa carenata con profilo rettilineo e breve orlo verticale" (Botto, 2009, 143145), databile fra la metà del VII sec. a.C. e la metà del secolo successivo. I frammenti esaminati sono in due casi di probabile produzione locale (SU63, 70), e di dubbia provenienza il restante esemplare. Due frammenti (SU112, SP127) sono identificabili con la tipologia della "coppa con carenatura alta e profilo curvilineo" (Botto, 2009, 136-138), rinvenuta nell'isola in contesti di metà VII - primo quarto del VI sec. a.C. Dei due esemplari, il fabric dell'orlo SU112 risulta accostabile ad una produ- 
SP39

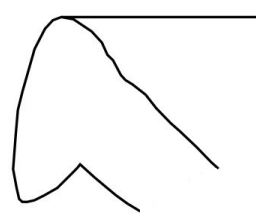

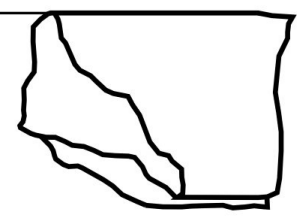

SP69
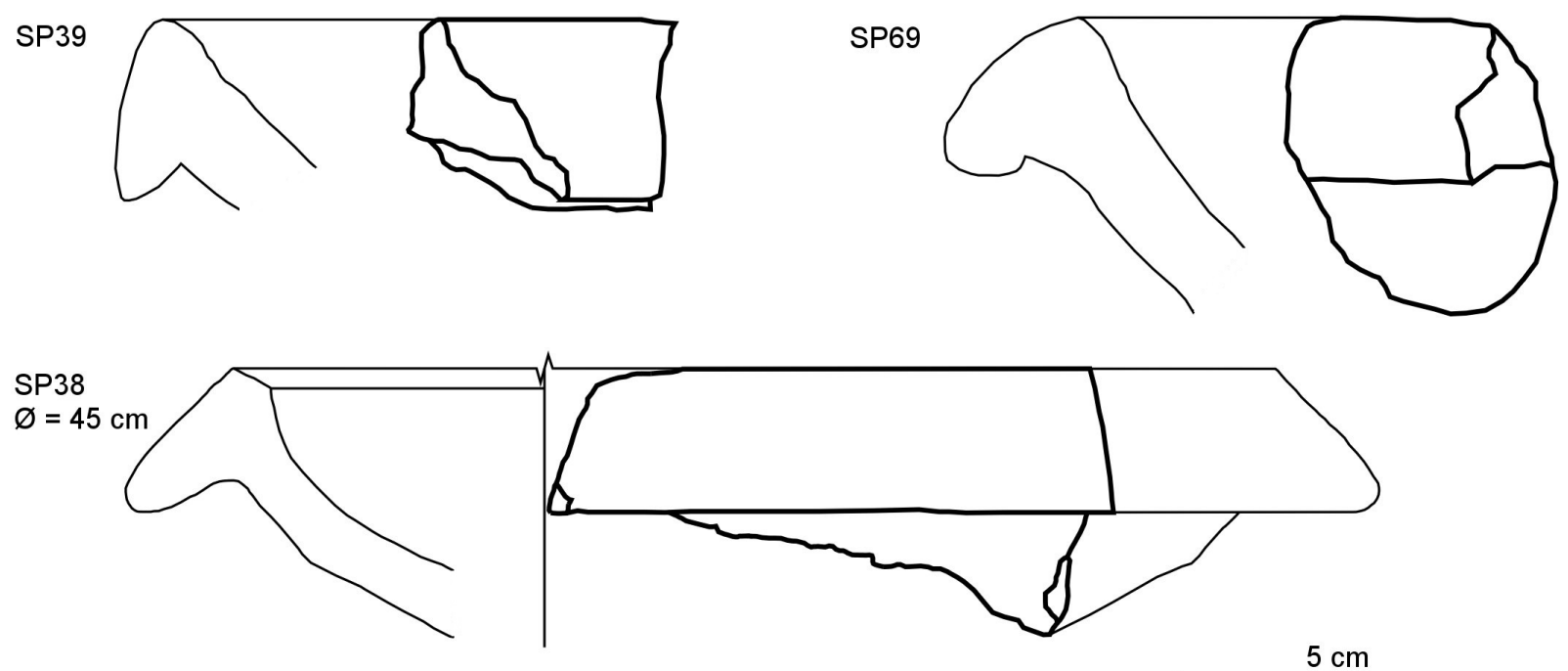

Figura 11. Disegni di frammenti di bacini.
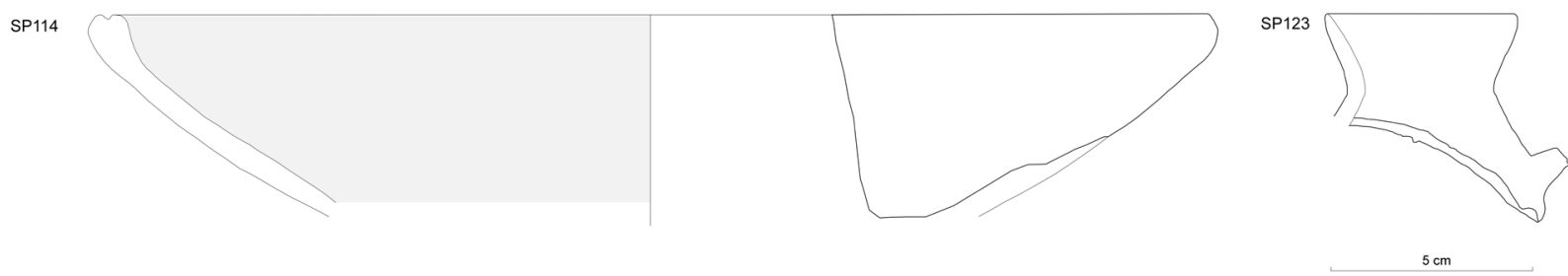

Figura. 12. Disegni del frammento di coppa SP114 e di attingitoio SP123.

zione nell'area del Sinis, di incerta attribuzione il frammento SP127. La tipologia della "coppa a calotta”, ampiamente diffusa in Sardegna (Botto, $2009,123)$ e le cui più antiche attestazioni in ambito indigeno provengono dal villaggio di Sant'Imbenia, nel nord dell'isola (Oggiano 2000, 243), è qui rappresentata dal solo frammento SU88, con fabric di difficile attribuzione e caratteristiche formali che orientano la cronologia dell'esemplare fra tardo VII e VI sec. a.C. (Balzano, 1999, 35, n. 134) (figura 13).

Orizzonti cronologici più antichi sono testimoniati dai piatti, in particolare dai due frammenti SU120 e SP118, rapportabili alla tipologia del "piatto con breve orlo estroflesso", databile fra la metà dell'VIII sec. a.C. e gli inizi del secolo successivo e diffusa sia in Sardegna (Botto, 2009, 101), che nel Mediterraneo centro-occidentale - ad esempio a Pithekoussai (Docter, 2000, 141, n. 8a). L'analisi dei fabrics dei due frammenti non ha permesso di identificare similarità con nessuno dei fabrics individuati (figura 14).

Leggermente successiva è la datazione del frammento di piatto SU87, probabilmente attribuibile ad un "piatto con ampio orlo estroflesso" (Botto, 2009, 104-105), inquadrabile nella prima metà del VII sec. a.C., e del frammento SU86, probabilmente parte di un piatto ombelicato databile nel corso della prima metà del VI sec. a.C. (Botto, 2009, 105-111).

La forma dell'attingitoio - dipper - risulta attestata con l'esemplare SP123, che trova un confronto con un esemplare rinvenuto nella necropoli di Bithia nella Sardegna meridionale e databile fra la metà del VII e la metà del VI sec. C. (Bartoloni, 1996, forma 21). Il fabric di questo esemplare appare accostabile al gruppo di provenienza tharrense.

Un frammento di orlo (SU22) di incerta produzione è attribuibile ad una brocca con bocca circolare che per le minute dimensioni è di difficile attribuzione tipologica specifica, ma probabil- 


\section{SU88}

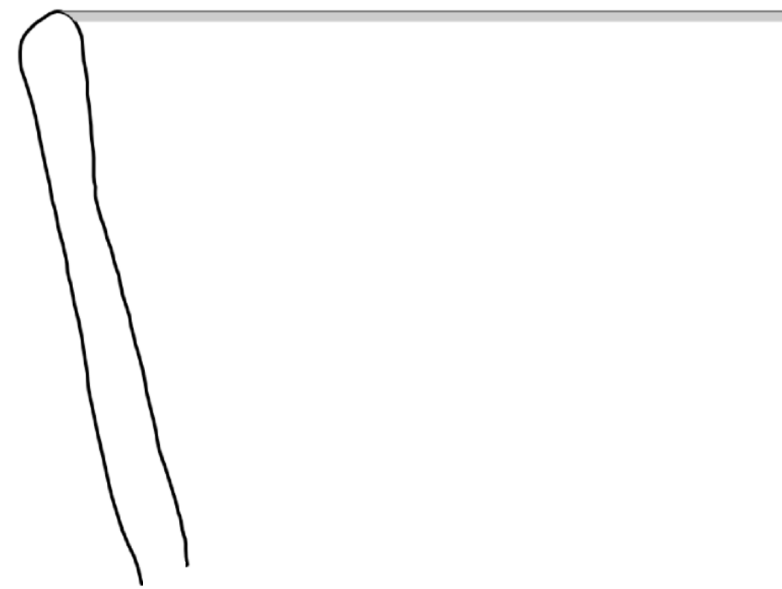

Figura 13. Disegno del frammento di coppa SU88.

mente accostabile alle "brocche/anfore con bocca circolare ed elemento divisorio sul collo", databili nella prima metà del VI sec. a.C. (Botto, 2009, 195-200)

\section{Discussione}

L'analisi dei materiali qui presentati permette di effettuare alcune osservazioni sia da un punto di vista diacronico sulla distribuzione cronologica dei materiali di tipologia fenicia, sia da un punto di vista qualitativo e funzionale in merito al tipo di ceramica fenicia presente presso nuraghe S'Urachi e l'adiacente sito di Su Padrigheddu.

\section{Aspetti cronologici}

Da un punto di vista cronologico, è ben osservabile un forte incremento di materiali fenici presso la comunità locale a partire dalla seconda metà - fine del VII sec. a.C., apprezzabile soprattutto prendendo in considerazione la datazione di anfo-

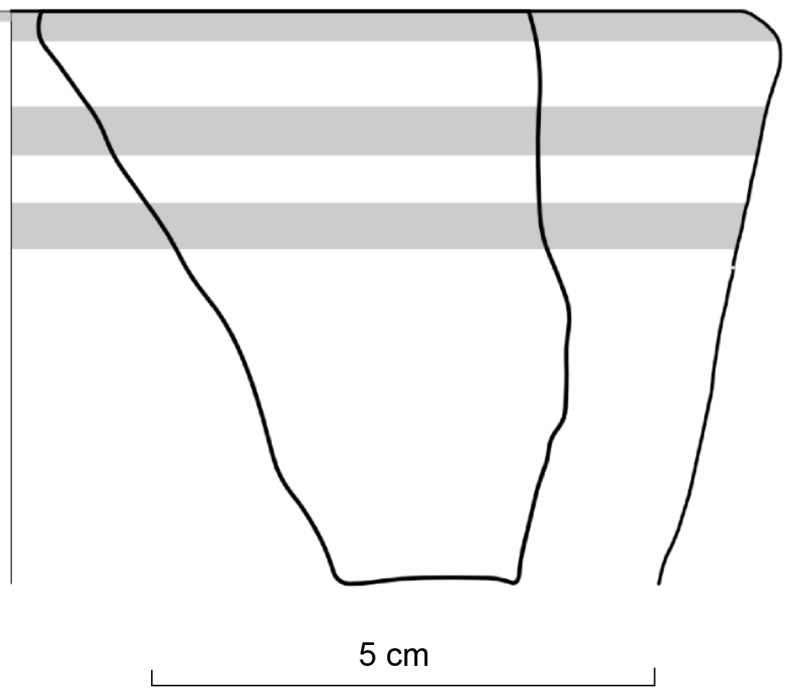

re, di alcune tipologie di cooking pots con copertura cronologica ridotta e della ceramica da mensa, mentre in generale i bacini e alcuni tipi di pentole per l'ampio spettro cronologico, che spesso abbraccia anche due secoli, non sono molto utili per una precisa scansione diacronica. Mentre i pochi reperti con cronologia alta, come il frammento di anfora tipo T-3.1.1.1. SU97 e i due frammenti di piatti con breve orlo estroflesso SU120 e SP118, testimoniano una limitata circolazione di materiali fra la seconda metà dell'VIII sec. a.C. e la metà del secolo successivo, analizzando tipologia e numero di esemplari dei contenitori anforici, spicca l'aumento di tipi fra la fine del VII sec. a.C. e il secolo successivo, fenomeno testimoniato in modo molto evidente dal numero degli esemplari riconducibili al tipo Ramón $\mathrm{T}-2.1 .1 .2$. = Bartoloni $\mathrm{B} 7$, numero che è più che raddoppiato nel corso del VI sec. a.C. considerando i tipi Ramón T-1.2.1.1. = Bartoloni D1 e Ramón T-1.4.2.1. = Bartoloni D2-3.
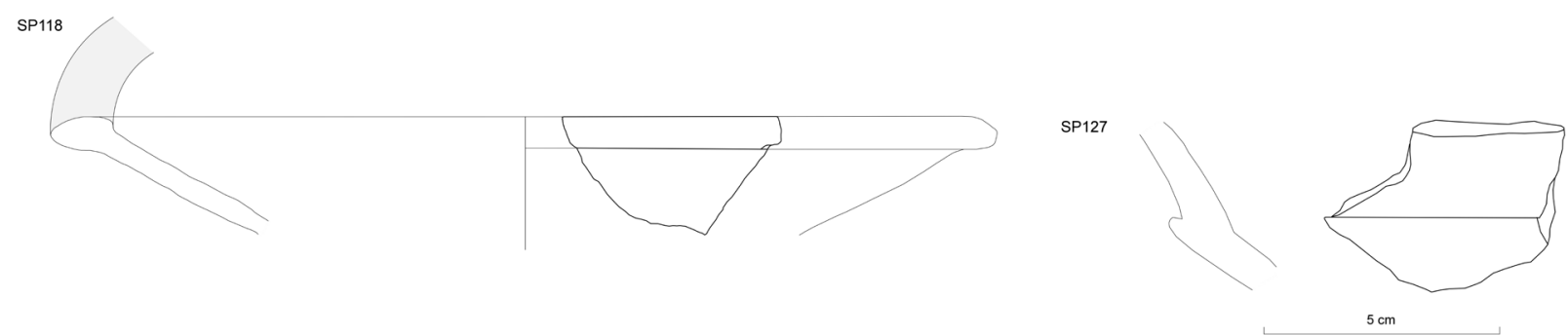

Figura 14. Disegni dei frammenti di piatto SP118 e di coppa SP127. 
Tale tendenza risulta confermata dall'analisi di tutti i materiali, come mostra il grafico (fig. 15), dove si nota un deciso aumento verso la fine del VII sec. a.C. - inizi VI sec. a.C. Mentre il picco in corrispondenza del volgere dei due secoli è certamente falsato dalla sovrapposizione di alcune fra le tipologie numericamente più rappresentate e a lunga copertura cronologica, come le anfore di tipo T-2.1.1.2., di tipo T-1.2.1.1., e di tipo T-1.4.2.1., è significativo che dai decenni finali del VII sec. a.C. si noti un deciso incremento nella distribuzione di materiali di tipologia non tradizionale. Questo fenomeno è particolarmente interessante se letto su base contestuale, a livello regionale: nel corso del VII sec. a.C. gruppi di Fenici si insediano presso il promontorio di capo San Marco a Tharros ed entro la fine del secolo a questo insediamento si aggiunge il centro fenicio di Othoca sulla sponda settentrionale del golfo di Oristano. A ciò evidentemente fa seguito un incremento dei rapporti commerciali fra queste comunità. Non a caso, i fabrics di gran parte delle anfore risultano compatibili con produzioni dell'area del Sinis dove era localizzato il centro coloniale fenicio di Tharros.

\section{Aspetti funzionali}

Queste osservazioni di carattere cronologico assumono maggiore interesse se si prende in considerazione il carattere funzionale dei manufatti, da un lato contenitori di trasporto come le anfore e ceramica spesso finemente prodotta per la mensa, e dall'altro forme funzionali alla preparazione e cottura dei cibi.

Le anfore fenicie testimoniano una richiesta da parte della comunità di S’Urachi e Su Padrigheddu di prodotti non locali, lavorati secondo la tradizione fenicia, come ad esempio potrebbe essere testimoniato dai resti di ovicaprini e bovini che sono ben attestati nell'isola proprio all'interno delle tipologie anforiche qui rappresentate (Finocchi, 2009, $386,406)$. V'è da ricordare che le anfore cosiddette da trasporto, tipologicamente realizzate secondo prototipi orientali, costituiscono una novità nel panorama ceramico nuragico, tant'è che un'innovazione tipologica e funzionale dell'età del Ferro è proprio la comparsa delle anfore tipo "Sant'Imbenia" - così denominate per la loro identificazione nell'omonimo sito indigeno nella Sardegna settentrionale (Oggiano, 2000) e presenti anche a S'Urachi/Su Padrigheddu) -, la cui produzione isolana è ormai attestata sulla base di analisi archeometriche (per la problematica relativa all'introduzione di queste anfore in Sardegna si veda Botto, 2011, 40-42; Roppa, 2012; Roppa, 2014 con bibliografia). Allo stesso modo, altre tipologie estranee alla tradizione nuragica, come alcune forme tra la ceramica da mensa, potrebbero essere state richieste dalla comunità locale per il loro valore intrinseco di

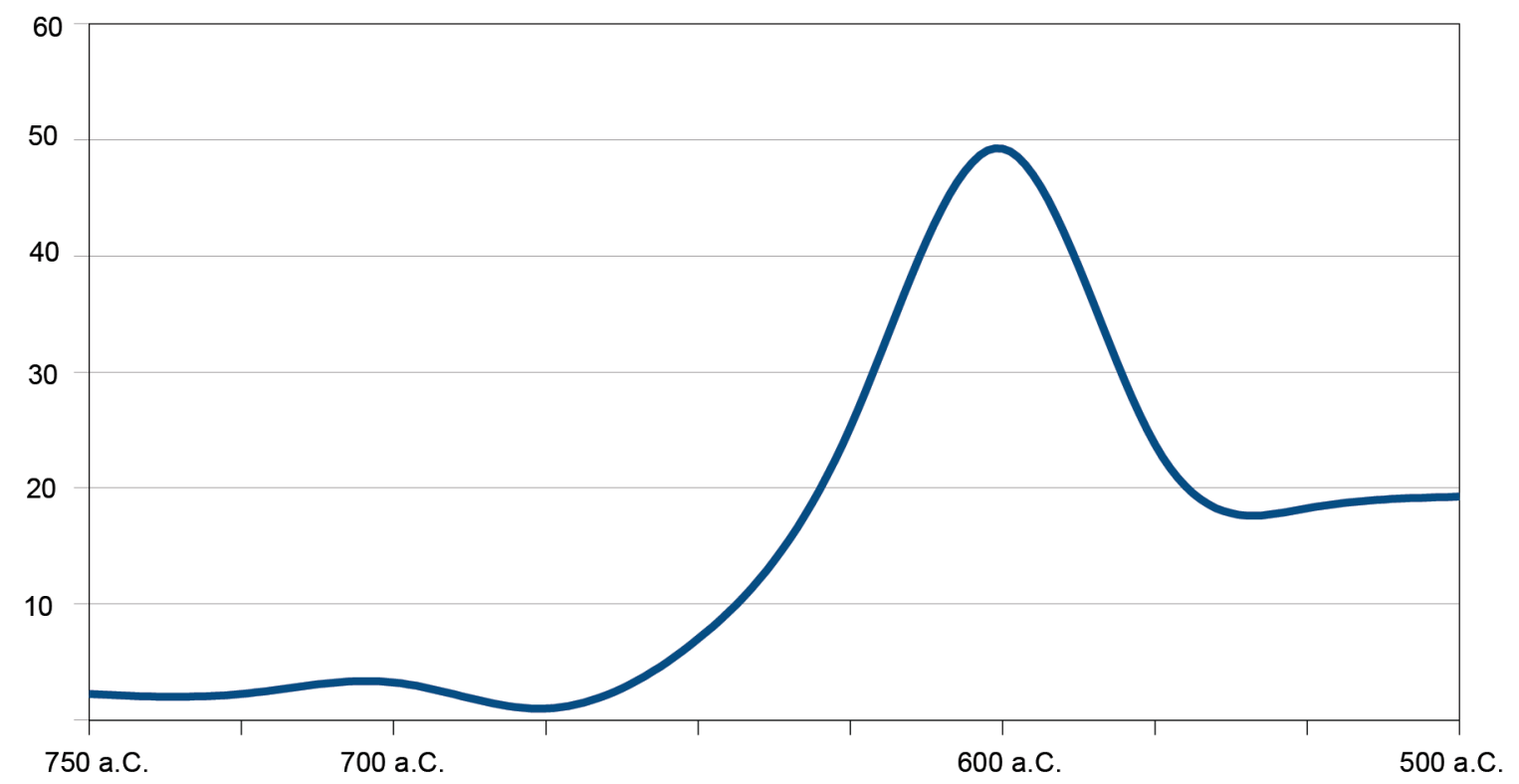

Fig. 15. Grafico della distribuzione cronologica dei materiali di tipologia fenicia, basato sulla media ponderata per cinquantennio. 
oggetto "esotico" ed essere state adeguate alle locali usanze di consumo - come potrebbe essere indicato dal numero ridotto di esemplari appartenenti a questa categoria funzionale.

$\mathrm{Da}$ un altro punto di vista, diverse indicazioni potrebbero essere fornite dalla ceramica da cucina e da preparazione. In questo insieme, infatti, sono maggioritari i fabrics di produzione locale che testimoniano una produzione in loco di questi manufatti per i bisogni di parte della comunità insediata a S'Urachi e nell'adiacente villaggio. Bacini e cooking pots sono delle forme connesse ad un tipo di preparazione dei cibi che probabilmente era parte del bagaglio tradizionale fenicio. Come è stato indicato da altri lavori sul mondo coloniale del Mediterraneo occidentale dell'età del Ferro (ad esempio Delgado e Ferrer, 2007), lo studio della ceramica da cucina può fornire importanti indicazioni sulle interazioni fra gruppi umani di diverse estrazione socio-culturale, in quanto è intimamente connessa ad una delle attività umane più distintivamente tradizionale, quale la modalità di alimentazione. Nel caso di S'Urachi/Su Padrigheddu, la diffusione di ceramica da cucina di tipologia fenicia prodotta in situ a partire dalla seconda parte del VII sec. a.C. può essere interpretata come una spia del progressivo insediamento presso la comunità locale di gruppi di background fenicio. Un'incrementata interazione fra gruppi di diverse tradizioni culturali e fenomeni di co-residenza a S’Urachi sono state evidenziate in altra sede anche sulla base dell'analisi del processo di produzione ceramica dei materiali di S’Urachi e Su Padrigheddu (Roppa, 2012; Roppa, Hayne e Madrigali, 2013), fra cui proprio i materiali da cucina di tipologia fenicia compresi nel gruppo di fabrics 2 e prodotte localmente appaiono nella maggioranza dei casi modellati attraverso delle tecniche di manifattura estranee alla tradizione nuragica e proprie invece della tradizione fenicia.

\section{ConCLUSIONI}

L'esame dei manufatti di tipologia fenicia provenienti da nuraghe S'Urachi e dall'adiacente villaggio di Su Padrigheddu permette di cogliere le vivaci dinamiche che attraversarono la Sardegna centro-occidentale nel corso dell'età del Ferro. Queste dinamiche, in un primo momento limitate alla circolazione di merci e al loro utilizzo da parte della locale comunità nuragica, assunsero maggiore complessità a partire dalla metà del VII sec. a.C., quando la presenza fenicia si fece maggiormente si- gnificativa a livello regionale. Molto probabilmente si verificarono dei modesti movimenti migratori di gruppi fenici che dai centri coloniali si insediarono nel grande centro sardo, innescando una serie di interazioni culturali che portarono grandi cambiamenti nel sito, cambiamenti pienamente maturati nel corso della successiva età punica, quando S'Urachi assunse delle caratteristiche che a livello di cultura materiale richiamano quelle dei grossi centri rurali che distinguono le campagne dell'isola e del contemporaneo occidente mediterraneo (van Dommelen e Gómez Bellard, 2008, 208-209; Roppa, 2013, 98-100).

Bibliografia

Atzori, G. (1992), “Il villaggio nuragico di Sant'Elia in Santa Giusta (Oristano)", La Sardegna nel Mediterraneo tra il bronzo medio e il bronzo recente (XVI-XIII sec.a.C.). Atti del III Convegno di studi "Un millennio di relazioni tra la Sardegna e i paesi del Mediterraneo", Selargius, 19 - 22 novembre 1987 ( Lilliu, G., Ugas, G. e Lai, G. Eds.), Cagliari, 127-134.

Balzano, G. (1999), Ceramica fenicia di Monte Sirai. Le forme aperte del vano C 33, Rivista di Studi Fenici Supplemento 27, Roma.

Bartoloni, P. (1988), Le anfore fenicie e puniche di Sardegna, Roma.

Bartoloni, P. (1996), La necropoli di Bitia-I, Collezione di Studi Fenici, 38, Roma.

Bedini, A., Tronchetti, C., Ugas, G., Zucca, R. (2012), Giganti di pietra. Monte Prama. L'Heroon che cambia la storia della Sardegna e del Mediterraneo, Cagliari.

Bernardini, P. (1997), "Le indagini nel settore occidentale del colle di Su Muru Mannu", Phoinikes B Shrdn. I Fenici in Sardegna. Nuove acquisizioni (Catalogo della Mostra di Oristano) (Bernardini, P., D’Oriano, R., Spanu, P. G. Eds.), Oristano, 124-126.

Bernardini, P. (2000), "I Fenici nel Sulcis: la necropoli di S. Giorgio di Portoscuso e l'insediamento del Cronicario di S. Antioco", La ceramica fenicia di Sardegna. Dati, problematiche, confronti. Atti del Primo Congresso Internazionale Sulcitano, Sant'Antioco, 1921 settembre 1997, Collezione di Studi Fenici, 38 (Bartoloni, P., Campanella, L. Eds.), Roma, 29-61.

Bernardini, P. (2005), "Neapolis e la regione fenicia del golfo di Oristano”, Splendidissima Civitas 
Neapolitanorum (Zucca, R. Ed.), Roma, 67124.

Botto, M. (2009), "La ceramica da mensa e da dispensa fenicia e punica", Nora. Il foro romano. Storia di un'area urbana dall'età fenicia alla tarda antichità 1997-2006. Vol. II - I materiali (Bonetto, J., Falezza, G., Ghiotto., A. R. Eds.), Padova, 97-238.

Botto, M. (2011), "Interscambi e interazioni culturali fra Sardegna e Penisola Iberica durante i secoli iniziali del I millennio a.C.”, Fenicios en Tartesos: Nuevas Perpectivas (Álvarez Martí Aguilar, J.), Oxford, 33-67.

Botto, M., Deriu, A., Negri, D., Oddone, M., Segnan, R., Trojsi, G. (2005), "Caratterizzazione di anfore fenicie e puniche mediante analisi archeometriche”, Mediterranea, 2, 57-106.

Campanella, L. (2009a), "La ceramica da cucina fenicia e punica", Nora. Il foro romano. Storia di un'area urbana dall'età fenicia alla tarda antichità 1997-2006. Vol. II - I materiali (Bonetto, J., Falezza, G., Ghiotto., A. R. Eds.), Padova, 295-358.

Campanella, L. (2009b), "La ceramica da preparazione fenicia e punica" Nora. Il foro romano. Storia di un'area urbana dall'età fenicia alla tarda antichità 1997-2006. Vol. II - I materiali (Bonetto, J., Falezza, G., Ghiotto., A. R. Eds.), Padova, 247-294.

Contu, E. (1952), "La fortezza nuragica di nuraghe Orrubiu presso Orroli (Nuoro)", Studi Sardi, 10-11, 121-160.

Delgado, A. e Ferrer, M. (2007), "Cultural Contacts in Colonial Settings: The Construction of New Identities in Phoenician Settlements of the Western Mediterranean", Stanford Journal of Archaeology, 5, 18-42.

Docter, R. (2000), "Pottery, Graves and Ritual I: Phoenicians of the First Generation in $\mathrm{Pi}$ thekoussai", La ceramica fenicia di Sardegna. Dati, problematiche, confronti. Atti del Primo Congresso Internazionale Sulcitano, Sant'Antioco, 19-21 settembre 1997, Collezione di Studi Fenici, 38 (Bartoloni, P., Campanella, L. Eds.), Roma, 135-149.

Finocchi, S. (2009), "Le anfore fenicie e puniche", Nora. Il foro romano. Storia di un'area urbana dall'età fenicia alla tarda antichità 1997-2006. Vol. II - I materiali (Bonetto, J., Falezza, G., Ghiotto., A. R. Eds.), Padova, 373468.
Hayne, J., Madrigali, E., Roppa, A. (c.s.), "Continuità e innovazioni formali nei materiali da S'Urachi: un riflesso di interazione tra Nuragici e Fenici”, L'Africa romana XX. Atti del convegno di studio, Roma.

Lilliu, G. (1950), "Scoperte e scavi di antichità fattisi in Sardegna durante gli anni 1948 e 1949”, Studi Sardi, 9 (1949): 399-406.

Lilliu, G. (2002), La civiltà preistorica e nuragica in Sardegna, Memorie della Accademia Nazionale dei Lincei, Classe di Scienze Morali, Storiche e Filologiche, Serie IX, Volume XV, Fascicolo 3, Roma.

Montis, I. (2004), "Il tofet di Sulcis: le urne dello scavo 1995”, Quaderni della Soprintendenza archeologica per le province di Cagliari $e$ Oristano, 21, 57-93.

Nieddu, G. e Zucca, R. (1991), Othoca. Una città sulla laguna, Oristano.

Oggiano, I. (2000), "La ceramica fenicia di S. Imbenia (Alghero-SS)", La ceramica fenicia di Sardegna. Dati, problematiche, confronti. Atti del Primo Congresso Internazionale Sulcitano, Sant'Antioco, 19-21 settembre 1997, Collezione di Studi Fenici, 38 (Bartoloni, P., Campanella, L. Eds.), Roma, 235-258

Ramón Torres, J. (1995), Las anforas fenicio-punicas del Mediterraneo central y occidental, Colleció Instrumenta, 2, Barcelona.

Roppa, A. (2012). "L'età del Ferro nella Sardegna centro-occidentale. Il villaggio di Su Padrigheddu, San Vero Milis", The Journal of Fasti Online, http://www.fastionline.org/docs/ FOLDER-it-2012-252.pdf

Roppa, A. (2013), Comunità urbane e rurali nella Sardegna punica di età ellenistica, Sagvntvm Extra, 14, València.

Roppa, A. (2014), "Manifattura ceramica, interazioni e condivisioni artigianali nell'età del Ferro sarda: i materiali da S'Uraki-Su Padrigheddu (San Vero Milis)", Materiali e contesti dell'età del Ferro sarda, Rivista di Studi Fenici, 41 (Roppa, A., van Dommelen, D. Eds.), Roma, 191-200.

Roppa, A., Hayne, J., Madrigali, E. (2013), "Interazioni artigianali e sviluppi della manifattura ceramica locale a S'Uraki (Sardegna) fra la prima età del Ferro e il periodo punico”, Saguntum, 45, 115-137.

Sebis, S. (1987), "Ricerche archeologiche nel Sinis centromeridionale. Nuove acquisizioni di età 
nuragica”, La Sardegna nel Mediterraneo tra il secondo e il primo millennio a.C. Atti del II Convegno di Studi "Un millennio di relazioni fra la Sardegna e i paesi del Mediterraneo", Selargius, 27-30 novembre 1986 (Lilliu, G., Ugas, G., Lai, G. Eds.), Cagliari, 107-116.

Sebis, S. (1998), "Il Sinis in età nuragica e gli aspetti della produzione ceramica", La ceramica racconta la storia. Atti del II Convegno di Studi "La ceramica nel Sinis dal Neolitico ai giorni nostri”, Oristano-Cabras, 25-26 Ottobre 1996 (Cossu, C. Melis, R. Eds.), Cagliari, 107174.

Stiglitz, A. (2007), "Fenici e Nuragici nell'entroterra tharrense", Sardinia, Corsica et Baleares antiquae, 5, 87-98.

Taramelli, A. (1935), Edizione archeologica della carta d'Italia al 100.000, Foglio 205 (Capo Mannu) e Foglio 206 (Macomer), Sassari.

Tore, G. (1984), "Per una rilettura del complesso nuragico di S’Uraki, loc. Su Pardu S. Vero Milis - Oristano (Sardegna)", The Deya Conference of Prehistory. Early Settlement in the Western Mediterranean Islands and their Peripheral Areas ( Waldren, W. H., Chapman, R., Leithwaite, J. E Kennard, R.-C. Eds.), Oxford, 703-723.

Tronchetti, C. e van Dommelen, P. (2005), "Entangled Objects and Hybrid Practices: Colonial Contacts and Elite Connections at Monte Prama, Sardinia", Journal of Mediterranean Archaeology, 18.2, 183-208.

van Dommelen, P. e Gómez-Bellard, C. (2008), Rural landscapes of the Punic World, London.

van Dommelen, P.e Trapichler, M.(2011). Fabrics of Western Sardinia. FACEM (version 06/06/2011) (http://www.facem.at/project-papers.php). 